\title{
E-Torch: A Mobile Commerce Location-Based Promotion System
}

\author{
Pantea.Keikhosrokiani \\ School of Computer Sciences, Universiti Sains Malaysia, \\ 11800 USM, Penang, Malaysia \\ Member, IEEE \\ Email: pantea.kia@gmail.com \\ Norlia.Mustaffa \\ School of Computer Sciences, Universiti Sains Malaysia, \\ 11800 USM, Penang, Malaysia \\ Muhammad Imran Sarwar \\ National Advance IPv6 Centre (NAv6), \\ Universiti Sains Malaysia, Penang, Malaysia \\ Nasriah Zakaria \\ Medical Education Department, College of Medicine, \\ King Saud University, Riyadh
}

\begin{abstract}
Mobile infrastructure technological development in the last decade leads to novel mobile commerce based advertisement addressing the businesses and end-consumers. Although location-based services developed rapidly in recent years, the reasons why we proposed a new system were lack of an intelligent location-based promotion system. In order to get the opinion and interest of users about modern promotion system, we conducted a preliminary study. Based on the preliminary study, we propose an e-Torch $\mathrm{m}$-commerce based mobile promotion system to facilitate businesses (such as hotel, restaurant, shopping mall etc.) and to advertise their products in an efficient way to the mobile customers according to their current Global Positioning System (GPS) co-ordinates. A proposed Ellipse model in this research assist the points of interest searching for mobile user according to current GPS co-ordinates by sending resultant information via cellular network (GPRS/3G). Furthermore, we justified proposed research by conducting a comparative study of the existing m-commerce based location tracking systems. Finally, we conducted experiments using simulation between notebook as client and remote server. We set clients' positions and used $3 \mathrm{G}$ usb modem for the data transmission. Round trip time was calculated to show success of the proposed system in different situations (indoor, outdoor, static and mobility).
\end{abstract}

Keywords: Mobile application, Mobile Commerce (M-Commerce), Location-Based Services, Global Positioning System (GPS).

\section{Introduction}

Attracting and satisfying the customers becomes more important than producing the goods in every business, as customers are the center point of each business. Therefore, advertisement plays an essential role in marketing activity in the modern era of market. In order to increase market share, sellers need to persuade potential customers to buy their product and services. Hence, effective advertising can increase consumption of a company's products. '1, 2 In addition, these days' customers are bombarded by many different product choices and confusion often occurs. An intelligent advertisement system is needed to assist the customer for making purchase decision. Advertisement will have effect on customer's attitude and buying behavior. 
Advertisement can be used in various forms such as mass media (TV, radio, newspaper and magazine) or it can be modern and intelligent advertisement system using mobile and Global Positioning System (GPS). In Mobile Commerce (M-Commerce) based advertisement systems, information on point of interests (e.g. hotels, restaurants, shopping malls, public and private sector venues), are needed, as well as any relevant promotional marketing packages. GPS technology is increasingly being utilized to enable customers to access services provided by modern advertising systems according to their current location. ${ }^{1,2}$

After launching GPS, first it was using in military applications in the early $1960 \mathrm{~s}$, but later its use has spread to civilian applications and services by the 2000s. As GPS signals can be received anywhere in the world without any distortion and within one meter of precision, it has advantage to other location tracking technology. ${ }^{3}$ We can call Garmin ${ }^{4}$ and Tom Tom ${ }^{5}$ among the first developers who developed applications used by GPS enabled devices that proposed a wide variety of services such as road navigation to weather and traffic update information as well as non-road information of potential interest to travelers, such as food and lodging. Foursquare ${ }^{6}$ made use of combination of location-based information and social networking to guide customers. Dunkin Donuts and Cold Stone Creamery are two more examples of sellers who used GPS-based advertisement services by using in-car GPS devices to inform drivers about special offers near their location. ${ }^{7}$ However, all of these developers (Garmin, Tom Tom, Foursquare, Dunkin Donuts, and Cold Stone Creamery) used location-based services to attract more customers; they did not focus on the location-based promotion systems.

The main problem that we would like to solve is customers' confusion among different choices and businesses (such as restaurants, hotels, etc) needs for attracting more customers. Thus, we propose an intelligent promotion system that can track the location of the customers and send the related promotions to them according to their current locations. This is the main goal of our proposed location-based promotion system.

Section 2 of this paper reviews location tracking from a technological perspective. Section 3 describes the impact of location-based service in M-Commerce as well as some related location-based services and systems. Section 4 presents a proposed e-Torch framework for M-Commerce, followed by results of experiments and discussion along with conclusion.

\section{Location Tracking}

\subsection{Global Positioning System (GPS)}

After six thousand years of searching for a navigation method for remote destination, Polynesians and modern navies utilized angular measurement of the stars for navigation. Later on, another type of navigation has been established by development of radio beacons. After the U.S Navi has developed all Navigation Satellite System in the 1960s and an artificial satellite called NAVSTARs was born in 1973. The current Global Positioning System (GPS), is a satellite-based navigation system created by U.S Department of Defense (DoD) to accomplish U.S military needs and later it became available for civilians. ${ }^{8,9}$ Nowadays GPS can offer positioning and timing information; it can be combined with map or GIS data to show the location in all around the world with any weather condition. ${ }^{9,10}$

\subsection{GPS-Based Location-Tracking}

GPS uses triangulation to calculate the real-time location of its users and provide position and time data. A location-based service is a mixture of communication technology, satellite navigation and geo-information. Coordinates and navigational parameters are delivered to the user from three or four satellites to compute both two and three dimension positions. GPS is a core technology for location tracking and is free of charge. ${ }^{11}$, ${ }^{12}$ GPS uses a position location technique from wireless communication called "multilateration". Multilateration locates objects by sending a signal to multiple receivers at known location and calculating the differences in arrival times. ${ }^{13}$ GPS calculates the distance between a satellite and GPS device; the satellites send a message with accurate orbit data and the exact time of sending; the receiver receives this information and computes the time differentials of these messages to calculate the location. User location information is given in latitude and longitude coordinates. ${ }^{14}$

\subsection{Cellular Network based Location-Tracking}

In this method of positioning location, mobile station (MS) must be located accurately to compute wireless signals parameters from different access points that are received by mobile device. Cellular network based location- tracking system consists of network-based and handset-based methods. In network-based method, the positioning measurements are calculated by base stations (BSs) while in handset-based method MS will recognize signals delivered by $\mathrm{BSs}$ to calculate the position. ${ }^{13}$ 


\subsection{Wireless Network based Location-Tracking}

Wireless Positionig System (WAP) that is a location tracking method by using wireless technology has been improved since GPS had some weaknesses. WAP maybe provides less precise tracking information but the coverage is better than GPS as well as the power consumption is less. Furthermore, WAP enabled devices such as iPhones, Android phones, etc made users familiar with map and utilizing WAP became simple and common. Consequently, a new horizon to the positioning application has been opened by technical advance of WAP. ${ }^{15}$ Indoor positioning system called Wireless Local Area Network, or WLAN that use WLAN infrastructures along with several methods such as AOA (Angle of Arrival), TOA (Time of Arrival) and RSS (Received Signal Strength) methods to calculate positions. WLAN covers a limited distance using high frequency radio signals to send and receive data. A WLAN location-based system refers to mobile devices that rely on a relationship between the location of the device and signals transferred from WLAN access points. ${ }^{13}$

\subsection{Hybrid Technology based Location-Tracking}

Hybrid-positioning system is combination of handsetbase positioning system such as GPS and network-based positioning system such as WAP to estimate positioning information. ${ }^{13}$ A-GPS is a good example of hybrid positioning system. ${ }^{16}$ The hybrid positioning system can reduce the problems occurred in other positioning systems as well as improve the position estimation of nodes. Furthermore, this method can assist us to reduce the number of base stations for position estimation and it can combines TOA (Angle of Arrival), TDOA (Time Difference of Arrival), and AOA (Time of Arrival)methods to establish an accurate measurement of $3 \mathrm{D}$ positioning location. Another example of hybrid positioning system is reference ${ }^{17}$ that proposed an autonomous and co-operative working model for cars that would collect and estimate the traffic jam statistics from source to the destination for each subject car by using an inter-vehicle communication systems. The proposed method gathers statistical traffic data using inter-vehicle communication (Wi-Fi and GPS) in order to get the estimation for the arrival time to destination. In this method, different geographical regions are divided into areas and the system analyzes the estimated time taken by the car to pass from one point to another in that specific area. Information for the research method was gathered by using a mix of technologies: short communication network (IEEE 802.11/Wi-Fi) and GPS receiver. Their proposed method of traffic simulation employs IEEE802.11 and is implemented in NETSTREAM simulator and $\mathrm{C}++.{ }^{17}$

\section{Related Work}

\subsection{Location-Based Service (LBS)}

Location-based services can be defined as services that can be accessed by mobile network, and utilized the current location of the mobile device appropriately. Many industries used GPS to enhance their products and services such as automotive industries that used navigation systems for their produced cars. Locationbased services assist user to access to the information regarding to the current geographic area of the user. ${ }^{18-20}$ Additionally, location-based services make possible two way communication and interaction between customers and businesses. In this way, users will get information according to their needs and requirements. Location based services are a combination of information and telecommunication technologies including Web GIS, Mobile GIS, Mobile Internet, Spatial Database, Internet and mobile devices. Generally, location based services are composed of some components: a mobile device, a communication network, a positioning component, a service and application provider, and a data and content provider. Recently location-based services developed quickly and they have been used for different purposes such as location-based social network (LBSN). ${ }^{21}$

\subsection{Impact of Location-based Service in M-Commerce}

Number of consumers who are using different services of mobile device increased recently. Because of ubiquitous and affordability of mobile device, not only customers used mobile device as an entertainment, but also as an advertising device. Fast growth of wireless technology expands electronic commerce (e-commerce) to mobile commerce (m-commerce). ${ }^{22}$

Mobile commerce (generally referred to as $\mathrm{M}$ Commerce) is the ability to conduct transactions using a mobile device. This generally makes business processes more efficient. With today's rapid technological advances, especially in mobile technology, people can 
communicate easily, anytime and from any place. In fact, M-Commerce will be more beneficial for new business services, if it is used for appropriate targeting of potential audiences/customers. These days, delivering real-time news about businesses to potential customers via modern advertisement systems is critical to improving the use of the M-Commerce. M-Commerce can join with location-based services to provide advertisement and promotion information directed at potential customer based on their current location. ${ }^{23}$

Electronic Commerce (E-Commerce) is known as subset of E-Business deal with electronic transaction. ${ }^{24}$ Enhancement in internet, network technology and mobile personal devices improved Mobile E-Commerce called M-Commerce. M-Commerce is E-Commerce transaction through mobile terminals over a wireless network to make business more efficient. M-commerce that is mobile-based commerce is different from traditional commerce systems and it is more beneficial for new business services. M-Commerce can be combined with location-based services (LBS) where the actual location of the terminal is used in service provision. ${ }^{23}$ The combination of M-commerce and LBS support can be used as a marketing tool to improve E-Marketing which will communicate and deliver value to the customers by using information technology. On the other hand, it manages customer relationship to benefit organizations and stakeholders. ${ }^{24}$

\subsection{Different Location-Based Services}

In this section, we review the successful software products that are based on Global Positioning System (GPS) for mobile navigation used in the cars. This software can be used as a standalone device (portable navigational system) or can be installed in the smart phones with some attractive functionality like audible driving direction, text-to-speech (TTS), real-time traffic updates and point-of-search interests. According to Goodwin (2010), GPS based navigational systems are Garmin Nuvi, Motorola MotoNav, Tom Tom XL and Magellan RoadMate. ${ }^{5}$

\section{Garmin Nuvi 3790T}

To this date, Garmin Nuvi 3790T is one of the thinnest and lightest portable navigational systems with best overall design. Some of the key features of Garmin Nuvi 3790T are Bluetooth calling, text-to-speech, downloadable maps and advance function to facilitate user. One of the disadvantages of this device is its glossy screen that makes it difficult to see for the end user especially while driving in the day light and importantly the data traffic cannot be accessed while using the hand phone feature. ${ }^{5}$

\section{Motorola MotoNav TN765t}

MotoNav have impressive technological advance hardware capability with innovative design and friendly user interface. Some of the important features of Motorola MotoNav TN765t are text-to-speech, Bluetooth handsfree calling, free FM traffic reception etc. MotoNav have robust and superior features than its competitors but lacks of auto GPS efficiency in the dense urban areas and its MotoExtra feature data service is not always on. ${ }^{5}$

\section{TomTom XL 340 S}

TomTom is an automotive navigation system with software and separate unit. TomTom can help people to locate a direction to go from their current location to destination, to show predictable arrival time, traffic jam, and also to expose an accurate map using process mining technique. ${ }^{25}$

As one the main competitors of Garmin, TomTom XL $340 \mathrm{~S}$ has advance features like lane guidance, downloadable fuel prices, detail information of complex freeway interchange, text-to-speech facility, multilanguage support (English, French, Spanish etc.) that also includes celebrity. The bottom-line is that TomTom XL provides all the fancy features with premium price. Apart from the good features, TomTom have some characteristics that can put it on the downside from other related software products that includes larger size, bulky, limited portability while routing in a moving vehicle that introduces the overhead in efficiency. ${ }^{5}$

\section{Magellan RoadMate 1470}

Magellan RoadMate has OneTouch feature that will allow user most used accessed destinations and searches. The advance features of Magellan RoadMate are destination confirmation features, 4.7 inch touch screen, more maps and menus etc. The main edge of Magellan with its competitors is its healthy and efficient performance and ease to use menus at a very low price. The poor design cradle interface with power cable when 
mounting and unchangeable on-screen keyboard layout is the downside of this device. ${ }^{5}$

\section{Foursquare}

Foursquare which is a social networking tool used for mobile device, has been founded in 2009. It introduced a new type of mobile marketing called mobile social media. Afterward, some companies such as Google Latitude (2009) and Facebook Places (2010) followed these kind of mobile social media applications but Fouresuare is still a market leader among those applications. Checking-in at the certain place will allow one user to be aware of other users' location by using Foursquare. ${ }^{26}$ Therefore; users can check-in at different places like restaurant, offices, hotels, etc and share their their location within their social networks. Moreover, users can discover their proximitities to their favorite products. Foursquare not only can be used by cellphone, but also it is accessible through computer browser. It can connect users to their contacts using their phone's address books as well as facebook and twitter accounts. 27

\subsection{Different Location-Based Systems}

Rapid technological evaluation affects electronic commerce (e-commerce) by changing the way of shopping, booking and marketing. Location-aware shopping has been developed by ${ }^{28}$ in order to provide information of the customer's preferred vendors that are in their neighborhood. Furthermore, ${ }^{29}$ provides a dynamic service discovery mechanism that enables mobile users in a given coverage area to easily access available services that are provided by suppliers. In addition, ${ }^{30}$ designed an intelligent agent based hotel search and booking system. The system is agent-based to perform hotel-booking activities. The agent will check all of the hotels in terms of available facilities, price, customer experience, transportation etc and forward this information back to the user's mobile phone. Moreover, location-based services can provide the wide range of information related to the Points of Interest (POI) such as hotels, restaurant, tourism attraction and so on. This information can be offered based on the current location of the tourists who are looking for an appropriate POI. ${ }^{31,32}$ and ${ }^{33}$ proposed tourism location-based services. The last location-based services are advertisement. Businesses can detect near- by customers and send them their promotion and new product in order to attract them to their businesses. On the other hand, this service will benefit customers who are looking for their favorite product. While the customer is confused between different products, sending promotion and product information can draw their attention to the special business close to their current location. ${ }^{34}$ and ${ }^{35}$ are proposed for locationbased advertisement services.

\section{Proposed E-Torch Framework for M- Commerce}

In the following section, we will discuss about research problem followed by two main contributions of this research that are preliminary survey and research on eTorch framework. The aim of preliminary survey is to investigate the number of mobile GPS users as well as understanding the user opinion and interests based on the modern GPS based advertisement system for hotel, restaurant, shopping mall, technology promotion. Moreover, we would like to evaluate the estimation of affordable price for e-brochure.

Next part of this research is m-commerce based advertisement system framework that uses GPS technology to track the location of the mobile user and cellular network for sending the query to e-Torch server in order to get the point of interests that can be hotels, restaurant, UNESCO sights based on the current location of the mobile. In order to make tracking of the points of interest efficient, we propose ellipse model that divides specified geographical location into several predefined ellipse shapes that have radius based on number of point of interests like hotel, restaurant, UNESCO sights etc. The diameter of the radius depends upon the number of the points of interest presented in the ellipses. Each ellipse is identified by a unique ellipse ID based on different location of the city. In the proceeding section, we will discuss about e-Torch framework along with the ellipse ID in detail.

\subsection{Preliminary Research}

A preliminary survey was conducted to get the users' opinion and interest concerning modern promotion system. The modern promotion system in this research is a real-time and location-based marketing system to deliver preferred information such as hotel, food or shopping promotion with respect to current GPS 
location of the mobile user. The goal of the preliminary study was to understand user's preference of Points of Interests (POI) in a GPS-based promotion system that would provide information about hotel, restaurant or shopping mall promotions according to the current location of the mobile user via short message service short message service (SMS) or multimedia message service (MMS). In line with this intention, the survey was conducted in Penang, Malaysia from 26 October, 2010 that was focused on the people working in the industry and students studying in university. ${ }^{36}$

To analyze the data from our survey, we used SPSS (Statistical Package for the Social Sciences) version 15.0, which provides descriptive statistics such as frequencies, descriptive analysis etc. Since this is a preliminary study, we did not undertake advanced statistical analysis. Given our requirements, we analyzed all the questionnaires to find the percentage of GPS users and the frequency of their preferred points of interests. We further calculated the frequency of users who preferred to have the e-Brochure cost or less than RM 0.50. Results of the analysis are presented in the next section.

The survey was conducted in Penang, Malaysia focusing on cell phone users. This online survey includes 10 simple questions in order to investigate required data for survey objectives. From the total of 100 respondents, $21 \%$ are Malay followed by Indian $(12 \%)$, Chinese $(21 \%)$ and others $(46 \%) .2 \%$ of the respondents are below the age of $20,31 \%$ between 21 25 years old, 35\% between 26-30 years old, $21 \%$ between 31-35 years old, $8 \%$ between $36-40,2 \%$ between $41-45$ years old and $1 \%$ of the respondents are between $46-50$ years old. These results shows young group of people are interested to use GPS.

The most important part of the survey was the percentage of GPS users: about $54 \%$ as. These respondents were filtered and analysis conducted on the essential data that identifies the survey objectives that is GPS users' data. For this reason, questions related to GPS users (for instance, the reason for rejecting GPS and MMS) were not considered.

After filtering GPS users from all respondents, analysis was conducted on the questions pertinent to the main objectives of this survey. The first objective of this survey was to determine the percentage of GPS users. Therefore, we filtered the respondents that gave a positive answer to the question "Are you a GPS user?"
The next objective was to identify GPS user's preferred services and POIs. Another objective was to investigate the percentage of MMS users within the GPS users and the amount of money they would be willing to pay. Finally, we needed to understand whether GPS/MMS users would be comfortable to receiving promotional and advertising messages to hotels, restaurants, UNESCO sites, etc.

We categorized respondents as GPS users and nonGPS users. From this, we could estimate the number of end users potentially interested in e-Brochure. According to the result of survey, $54 \%$ of cell phone users also use GPS, meaning that $54 \%$ are likely to be interested. Next, we investigated their preferred services in order to identify the scope of e-Brochure. $85.2 \%$ of the GPS users are interested in map services; therefore, we will include a map search option as a part of our software menu. We will send promotions and advertisements related to points of interest to users, which we will display on the map relative to their current location, and we will navigate their selected users to the destination POI.

We also categorized respondents based on whether they preferred to use SMS or MMS, so we could determine how many users would able to receive POI information through SMS or MMS. According to our survey, a healthy number of GPS users (68\%) prefer to use MMS. Accordingly, we will focus on providing our location-based advertising information through MMS.

In the survey, a list of POIs has been given to GPS users and they were asked to select their preferred POIs based on their interests. The results were food promotion 54.3\%, shopping promotion $42.9 \%$ entertainment $45.7 \%$, life-style $22.9 \%$, hotel information $40 \%$, UNESCO sites $71.4 \%$, technology promotion $48.6 \%$, spa promotion $14.3 \%$ and monthly event updates $31.4 \%$. A high percentage of users are interested in promotions for both hotels and restaurants, which matches the focus of our research. UNESCO sites and food promotion garnered the highest percentages (71.4\% for UNESCO sight and $54.3 \%$ for food promotion). On the other hands, the first thing that all travelers will look for is a hotel. Consequently, we will also focus on the hotel as POI, despite its relatively low preference ranking.

We can reasonably conclude how much GPS users' are willing to pay for a location-based advertising service like e-Brochure. A majority of the respondents 
(76.9\%) stated they would be willing to pay less than 50 cents; only $23.1 \%$ would pay more than 50 cents. Finally, we have a better understanding of users' willingness to receive promotion and advertising messages. $75.9 \%$ of GPS users would agree to receive such messages. Consequently, we can feel comfortable about sending location-based promotion and advertising information regarding hotels, restaurants and UNESCO sites to users.

\subsection{Overview of Proposed E-Torch Framework}

Different mobile commerce based advertisement models is discussed and evaluated in detail in section 3. Various researches in previous sections assist us to evaluate the pros and cons of each model. Based on this study, we propose e-Torch mobile commerce based promotion system that uses GPS technology to track the location of the mobile user. Based on the GPS co-ordinates, the system can explore the unique ellipse ID to make an efficient search for receiving the list of point of interest based on that specified ellipse ID.

\section{3. e-Torch Framework Methodology}

This section will discuss about the research methodology, illustrated in Figure 4.23; its architecture, modeling and framework in detail. The research methodology phases are explained briefly in the proceeding section:

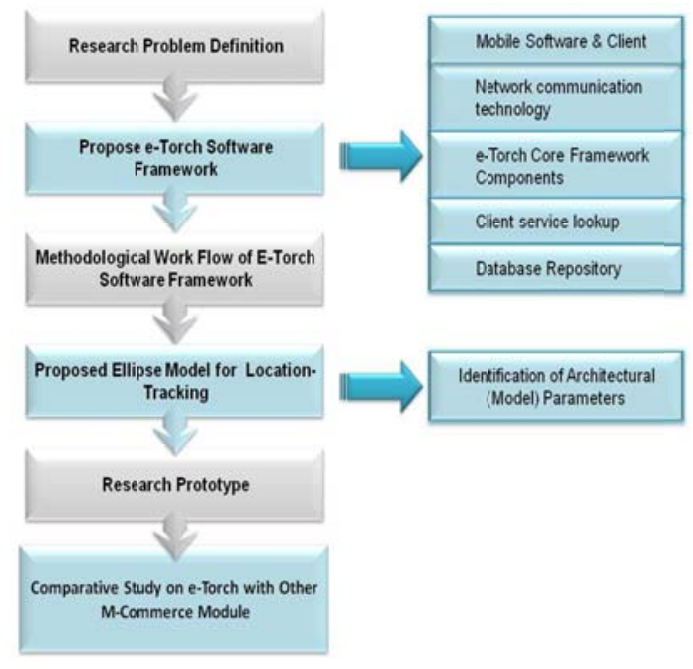

Fig. 1: Research Methodology for e-Torch Framework (source: developed for this study)

\section{Research Problem Definition}

In section 2 of this paper, we discuss different work related to communication technology used for tracking location of the mobile user along with mobile commerce used for advertisement. Based on these parameters, we critically review their proposed methods and highlighted the points that they failed to address. After thorough investigation in the previous section, we are able to identify our research problem definition.

In the mobile-commerce, tracking the location of the mobile user is important in order to provide location-based information of the points of interest (hotels, restaurant, UNESCO sights, shopping mall etc.) that are located near the user's geographical area. Normally, location tracking is done by the GPS (Global Positioning System) or A-GPS (Assisted-Global Positioning System) from the mobile device that provides the exact co-ordinates of the mobile user's location. Later, the location co-ordinates can be used by the company (providing the m-commerce services) to search for appropriate points of interests that have been requested by the user through wireless or cellular network. In section 2, we discussed different techniques to locate mobile users through GPS and A-GPS technology. Moreover, a discussion on different solutions and frameworks that have been used by the $\mathrm{m}$ commerce based companies to provide the appropriate point of interests back to users was presented. Apart from research innovations, some problems need to be addressed particularly. A modeling technique is needed that is able to accommodate and search multiple points of interests with accuracy, efficiency and robustness; therefore, the mobile user does not need to wait for response with much delay. Furthermore, there is a lack of framework that can handle multiple points of interest and its repository for $\mathrm{m}$-commerce based solutions especially in terms of promotions.

\section{Proposed e-Torch System Framework}

We propose hybrid cellular network and Global Positioning System (GPS) based mobile client system that navigates and tracks the location of mobile user to deliver accurate information in detail about hotels, restaurants, shopping malls and UNESCO sights to the user of the mobile device.

E-Torch framework will be a bridge between client and server to deliver location-based and real-time 
advertising message to users. The e-Torch framework architecture, illustrated in Figure 2, is composed of important abstract layers namely: Mobile Software and Clients layer, Network Communication Technology layer, e-Torch Core Framework Component, Client Service Lookup (such as UNESCO Site, Google Maps, Shopping Mall advertisements, Restaurant and Hotel lookup services respectively), and e-Torch Database Repository layer.

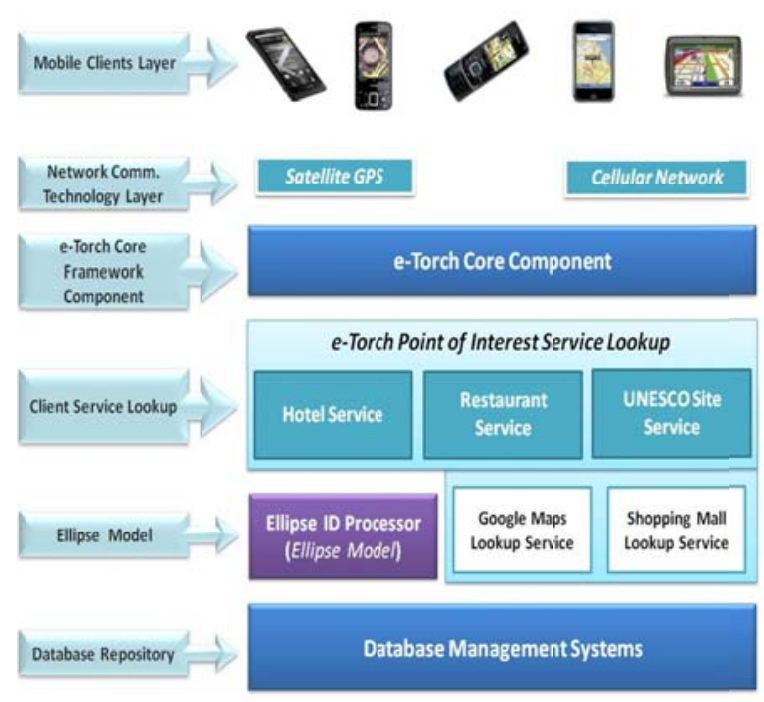

Fig. 2: Proposed e-Torch Framework for M-Commerce (source: developed for this study)

\section{Layer 1: Mobile Clients Layer}

Generally, GPS enabled mobile device communicates with satellite network to get its current GPS coordinates and then communicates with the remote server located in different geographical location (Wang, et al., 2010). Similarly, we propose e-Torch application that will run on the Android-based mobile GPS phones. The mobile device should be Java-enabled and have the capability of GPS in order to send correct navigational parameters to the e-Torch server. The maps can be downloaded from Google server for free and have the capability to mark the locations such as hotels, restaurants and UNESCO sights. The location tracking mechanism will be based on our proposed methodology especially by using ellipse model for efficient location tracking of mobile devices.

Users need to download free Java-based e-Torch software application and install it in their Android-based
GPS phone. Mobile device will track its own current location through GPS. Clients need to select their point of interest from e-Torch menu to receive related marketing information.

\section{Layer 2: Network Communication Technology Layer}

We propose to use a hybrid network communication technology that is a combination of GPS for location tracking and cellular network using GPRS/3.5G for the communication between the mobile device and remote server. The use of GPS will help the mobile device to get its current location co-ordinates that it will be sent to the e-Torch server by using cellular network to choose specific hotels, restaurants, UNESCO sights near to mobile device current location by using ellipse model for tracking. The location will be covered using ellipse area that will be given a unique ID. Based on that ID eTorch will able to search and send the information related to the hotels, restaurants, etc advertisement and promotion to the mobile user. The communication between the mobile device and the e-Torch server will be through e-Torch communication protocol that is optimized.

Cellular network is the bridge between e-Torch enabled mobile device and the e-Torch framework server. The mobile users must choose their preferred points of interest through e-Torch menu and send it to the server where the server needs to process the information through database repository in accordance to the current location of the mobile device and send the advertisement and promotion information back to mobile client.

\section{Layer 3: E-Torch core Framework Component}

E-Torch core framework component is the most important component that deals with different services lookup (such as UNESCO Site, Google Maps, Shopping Mall advertisements, Restaurant and Hotel lookup services respectively), as well as communication protocol between the mobile client and the e-Torch server by first taking the GPS co-ordinates of the mobile client and then process the preferred points of interest (POI) information. Moreover, it also acts as bridge between the server application and the database repository that in our case is mySQL database container. For the e-Torch administrator, the required information of the hotel, restaurant, and UNESCO sights will be inserted, updated, searched and deleted in the database. 
Importantly, the GPS location processing and searching for appropriate places will also be handled by this component. The e-Torch framework is handling the lookup service that is one of the most important component of the e-Torch framework that are UNESCO sight, Google maps, Restaurant information, hotel information etc. that is being processed with the help of e-Torch database repository layer.

Therefore, e-Torch core component is responsible for handling the communication layer of the e-Torch framework and other component of the server side. One of the important tasks of this layer is to de-encapsulate the message received from the mobile device, encapsulate the message received from the server side of e-Torch, and send it back to the mobile client. The communication protocol between the mobile device and the e-Torch server component will be based on XML (Extensible Markup Language) message based communication protocol.

\section{Layer 4: Client Service Lookup}

Client service lookup is an important component of the e-Torch framework that acts as a bridge between the eTorch core component and the database repository. Firstly, the service lookup component takes the GPS location parameter information of the client from the eTorch core component and then sends it to the database repository in order to get the corresponding ellipse ID of the geographical location. Then it will store the ellipse ID information and get the point of interest information that is been sent by the user to the e-Torch core component using e-Torch menu and forward it to the database repository. The database repository after processing the information will give back the result that will be forwarded to the client service lookup. Whether, the client request for the point of interest is one or more, all the relative information will be handled by the client service lookup. The information will be later forwarded to the e-Torch core component that will able to build the message in the XML format.

Consequently, this layer plays as a container to keep all the data related to the user interests and GPS locations and transfer them to Database Repository Layer. On the other hand, the results of searching in Database Repository will be saved in Service Lookup and then forwarded to the e-Torch core component.

\section{Layer 5: Ellipse Model}

The e-Torch framework will receive two parameters from the e-Torch user client that are GPS location coordinates and preferred point of interest (POI) such as restaurant, hotel and UNESCO sites respectively. The ellipse processor will get the GPS co-ordinates location, give it to the database management system that will search and return the unique ellipse ID in accordance to the user current location. The ellipse Model processor generates ellipse ID that is explained in detail in proposed ellipse model section of the research methodology. Furthermore, the return ellipse ID will be given to the database management system of e-Torch that will search for the appropriate point of interest for that specific location. The database management system will process the request and generate the list of point of interests that will be sent back to the user later.

\section{Layer 6: Database Repository Layer}

The final and bottom most layer of the e-Torch framework is the database repository layer that deals with user information as well as lookup services of GPS location, restaurant information, hotel information, UNESCO sight details based on the ellipse ID that is been retrieved and processed. The database repository component deals with the parameter query search that is sent by service lookup component based on the ellipse ID. It will process the requested information of point of interest (hotel, restaurant, UNESCO sights) and give advertisement and promotion result back to the service lookup component that will forward to the e-Torch core component. The e-Torch core component will build the message and send the result back to the mobile client through the cellular communication network.

Database Repository is storage place for all the data related to the points of interests, users, ellipses and GPS locations. The GPS location of the mobile device have been forwarded to the database repository by the eTorch core component and based on the GPS locations the request point of interests will be searched in the Database repository and sent it to the Service lookup layer.

\section{Methodological Work Flow of e-Torch Software Framework}

Methodological workflow connects all e-Torch framework layers together and makes this framework 
efficient. The important components that have been identified in the methodological flow are mobile device, communication medium, e-Torch core component, GPS locator, Service Lookup and importantly the database manager component.

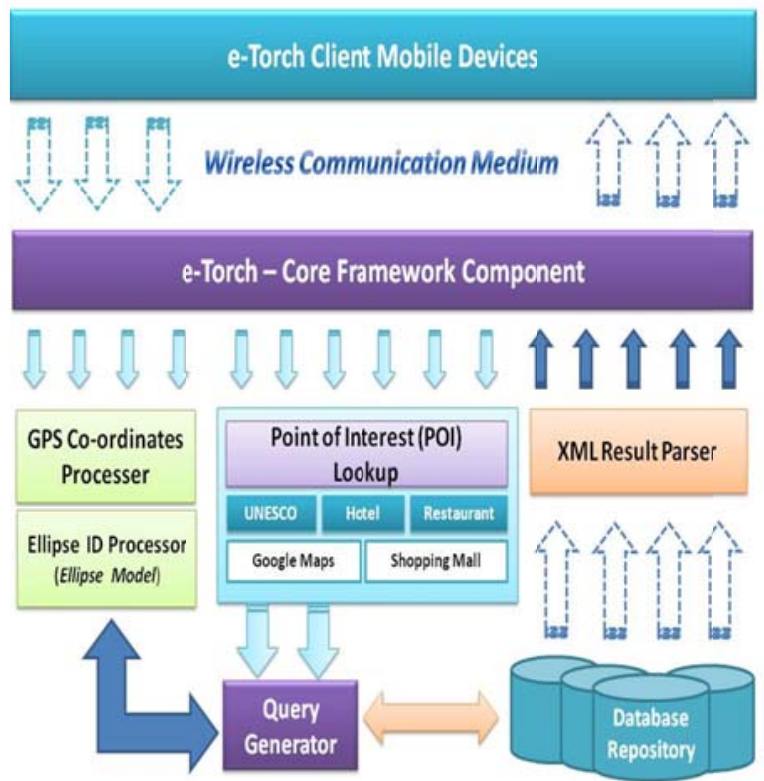

Fig. 3: Methodological Flow of e-Torch Framework (source: developed for this study)

In the workflow, as shown in Figure 3, client has already installed e-Torch software application in their Android-based mobile phone. Once the client opens the e-Torch application, the requested GPS location summary from Satellite will be stored in the mobile device. The main menu of the e-Torch framework is been displayed to the mobile user for the selection of the point of interest that in our application are Hotel, Restaurants and UNESCO sights etc. After the user selects the point of interest, the GPS location coordinates along with the point of interest is been sent to the e-Torch remote server through internet.

On the other hand, the e-Torch server receives the mobile client request that contains its current GPS coordinates along with required point of interests. The eTorch core component will receive the request of the mobile client and then disassemble (disperse, dismantle) the message and forward the GPS location co-ordinates to the GPS Co-ordinates Processor component of the eTorch framework.
The GPS Co-ordinates Processor will send the information related to the mobile user current location to the database in order to get the appropriate ellipse ID with respect to the mobile GPS location. The e-Torch core component will send the selected point of interest directly to the service lookup component that will make a query request based on the ellipse ID and send it to the database component. The database component manager will process the information and search the specified point of interest based on the ellipse ID that was the result of the GPS location send by the client. The result will be sent to the XML Result Parser that will forward to the e-Torch core component. The e-Torch core component will build the message that contains list of the point of interests and send it to the mobile client through cellular network. On receiving the information, the mobile device will display the information on the screen.

\section{Proposed Ellipse Model for Location Tracking}

After our comparative study of different location based services and their critical analysis, we propose ellipse model that address weakness left in previous work. The proposed ellipse model divides specified geographical area into several predefined ellipse shaped sections that have a radius based on the number of Point of Interests (e.g., hotels, restaurants, shopping malls, UNESCO sights etc.). The diameter of the ellipse is dynamic in nature that means if the point of interest are close together then the resultant diameter will be smaller and vice versa. Moreover, the GPS location co-ordinates will be incorporated with the ellipse ID making it faster for identifying the appropriate ellipse zone with respect to the location of mobile user. Hence, it is making the system of framework accurate, efficient and robust. The ellipse zone will only be presented in the geographical area where the point of interest will be located. The size of the ellipse zone will be based on the number of point of interests in that particular geographical area. The ellipse model will be defined only on necessary or important places where the points of interest lie.

The ellipse Model is a hierarchical model in which location of point of interests have been organized in tree-like data structure; for example, each country can have many cities, where each city can have many areas/town that have point of interest places like restaurant, hotels, UNESCO sights etc. The scope of this research is limited to Penang that has many tourist 
attractions such as UNESCO sights and accommodation for traveler such as hotels, restaurants and as point of interest for the users. Each town can have more than one ellipse IDs assigned to them on the basis of number of point of interest. The ellipse ID for Batu Ferringhi that is well known tourist spot will be created using country code, city code and area code as MAL-PEN-016 as explained in Figure 4.

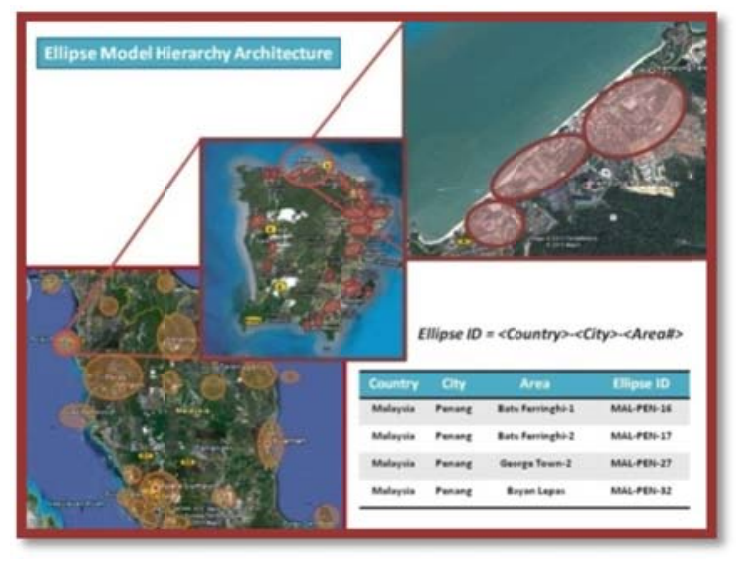

Fig. 4: Ellipse Model Hierarchy Architecture (source: developed for this study)

Mobile user sends the request of the job (hotel, restaurant etc.) along with the GPS co-ordinates to the e-Torch framework that will be later given to the eTorch repository to lookup the ellipse ID along with the list of hotels or restaurants that are presented in that specific ellipse. The diameter of the ellipse is dynamic in nature that depends upon the number of point of interests. For example, each ellipse can have maximum of ten points of interest from every categories such as hotels, restaurants and UNESCO sights. Therefore, maximum number of points of interest accommodated by each ellipse will be 30 as more points of interest can cause network congestion, add complexity and affects the quality of service. The GPS co-ordinates of the mobile device plays an integral role in getting ellipse ID that will be needed in the e-Torch framework to get the hotel, restaurant or UNESCO sight lists.

In e-Torch systems, points of interest that are hotels and restaurants have been identified on different location of the city. Then ellipse IDs have been mapped against the point of interests based on their number and importance. For example, the locations that have more hotels and restaurant have more ellipses. Later, the information of the hotels along with the restaurants has been added in the e-Torch repository corresponding to the ellipse ID. This is an efficient approach, as ellipse ID will be defined where the point of interest is present and the area without points of interest has no ellipses. When the mobile user request the point of interest to the e-Torch framework system, then the ellipse ID have been mapped according to the current GPS location of the mobile user and then its corresponding point of interest will be send to the mobile user.

The information will be retrieved from the e-Torch database, build as XML data format and send to the mobile user. On receiving the information, the data will be parsed and displayed on the e-Torch user interface that shows the map and labeled list of point of interests like restaurant, hotel, and shopping mall as shown in the Figure 5. If some of the new points of interests are not in Google map repository, we will add that point of interest in e-Torch defined ellipses in database.

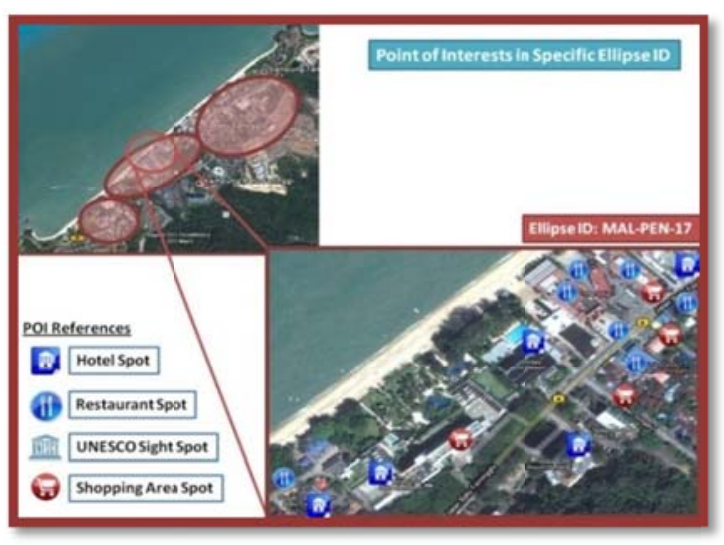

Fig. 5: List of Point of Interest for Specific Ellipse ID (source: developed for this study)

After receiving labeled list of point of interests illustrated in Figure 5, more data will be displayed on the e-Torch user interface that shows the list of the name of the hotels, restaurants, and UNESCO sights as shown in Figure 6. In case of points of interest (POI) that are subscribed to e-Torch, details of the promotion as well as their description will be displayed on the screen to the user. 


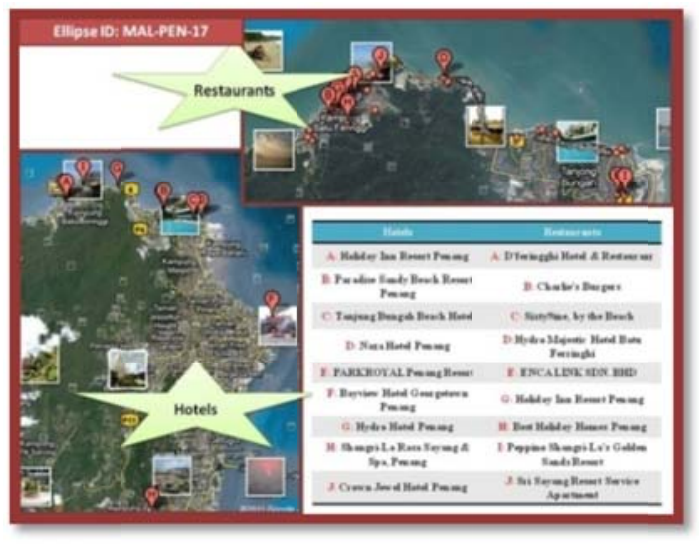

Fig. 6: List of Point of Interest in Detail for Specific Ellipse ID (source: developed for this study)

Figure 7 shows the user navigation directing user from its current location and the nearest Point of interest (POI) ellipse.

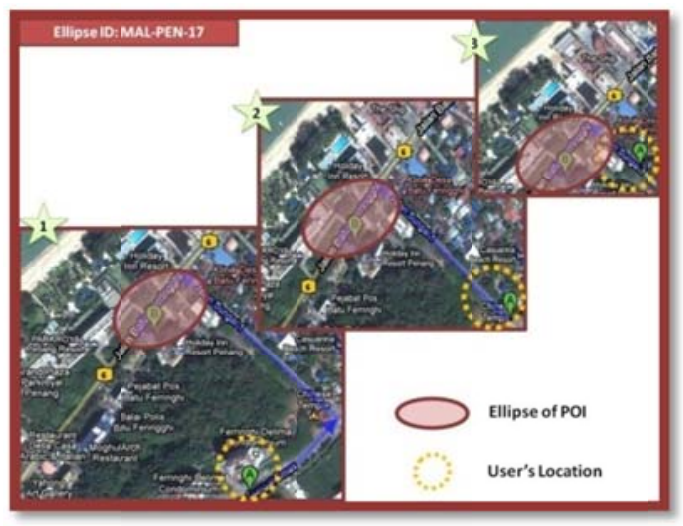

Fig. 7: User Navigation to Ellipse of POI (source: developed for this study)

Suppose the user open the e-Torch application and there are no points of interest in the user's current location. The e-Torch system will get the user location co-ordinates and draw a dotted circle in the map around the user current location. Moreover, the arrow path will be drawn from the user current location to the destination point of interest ellipse. In this way, e-Torch will direct the user to the nearest point of interest ellipse available with proper navigation system. The user will follow the path that will lead to the nearest point of interests that have hotel, restaurant or UNESCO sights until the user dotted circle overlap with the point of interest ellipse as shown in Figure 7.

\section{Identification of Architectural (Model) Parameters}

The main goal of this research is to deliver real-time and location-based information to the user to pick out the best selection. For this purpose according to the Figure 8 , the target geographical area is divided into predefined ellipses that have a radius based on the number of Point of Interests (e.g., hotels, restaurants, shopping malls, UNESCO sights etc.).

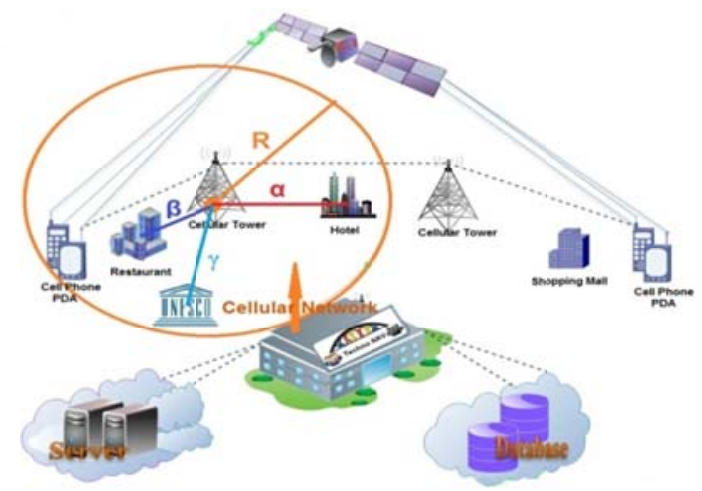

Fig. 8: Proposed Ellipse model Architecture for location tracking (source: developed for this study)

The ellipse zone will be helpful to search the points of interest in accordance to the location of the mobile user, as every ellipse zone is based on the location coordinates and contains the information of the point of interests. The area of ellipse zone depends on the number of point of interests and has five important parameters including $\mathrm{R}, \alpha, \beta, \gamma, \lambda$. $\alpha$ is space between center point of the ellipse zone and location of the hotel, $\beta$ is space between center point and location of the restaurant, $\gamma$ is related to the distance between UNESCO site and center point and eventually, $\lambda$ is space between center point and shopping mall. The distance of the point of interest parameters $\alpha, \beta, \gamma, \lambda$ should always be less than the radius of the ellipse zone otherwise, they will reside outside of the ellipse zone that is been mentioned in the equation below:

$$
\alpha, \beta, \gamma, \lambda \leq \mathrm{R}
$$

More ellipse model can be defined later in case of new UNESCO Sites, Shopping Malls, Restaurants and 
Hotels that resides outside of the current ellipse zone; therefore, ellipse zone will be less congested.

\section{Research Prototype}

In this section, we will discuss about the application process flow of the prospective from client and server. Figure 9 is a process diagram that explains the start of the e-Torch application process from the mobile device when it sends the parameters to the e-Torch server where it process the information and send it back to the mobile clients. The detail is explained below.

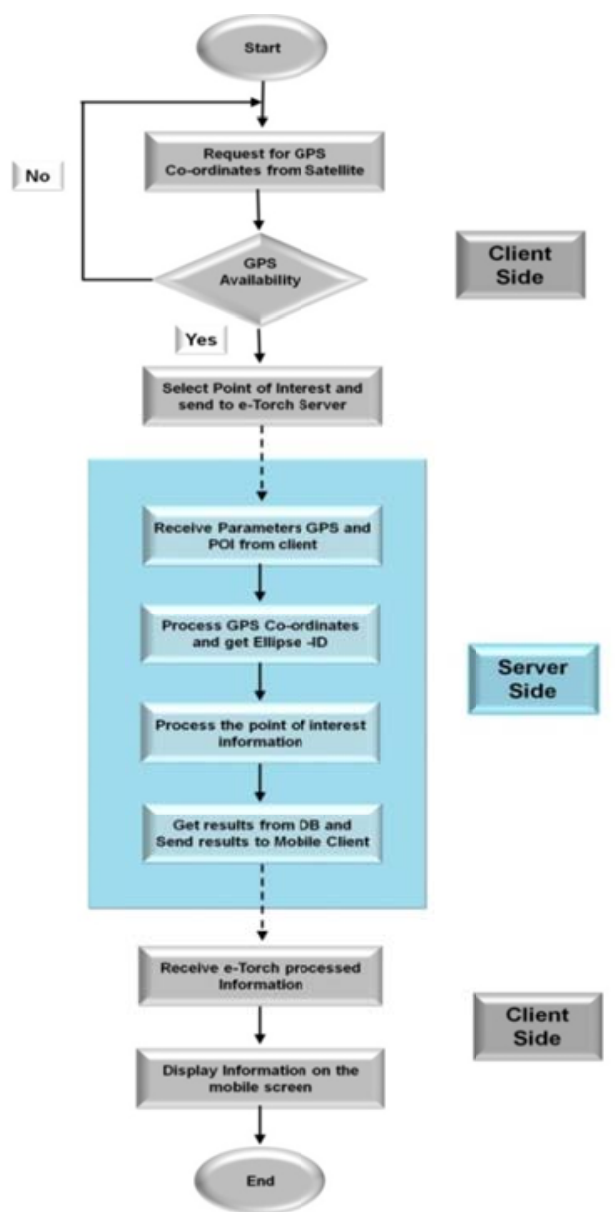

Fig. 9: Process flow of the e-Torch client and server application (source: developed for this study)

The e-Torch server will receive the request of the mobile client and able to grant the permission to connect with it and receive the GPS location coordinates as well as the point of interests that is been selected by the mobile user from the selection menu in the mobile device. It will then process the information of the GPS co-ordinates and based on those co-ordinates will get the ellipse-ID. Then the ellipse ID will be sent to the database repository in order to search for relevant point of interest selected by the user itself. The database repository will be able to execute the query and send the result back to the main component of the server. The server will send the result back to the mobile device that will able to display the resultant data on the mobile device. Hence, the job process is been successfully executed.

The implementation design of e-Torch is been divided into four phases that to make the components work together. The layers are:

- Graphical user interface (GUI) [server admin]

- Application Layer

- Database Layer

- Communication protocol

\section{Graphical User Interface (GUI)}

The mobile user of the e-Torch framework will be asked to submit the point of interest i.e., hotel, restaurant and UNESCO sights by the help of graphical user interface. E-Torch application software will be started by the splash screen that shows the logo of the e-Torch software and proceed to the e-Torch menu that gives the point of interest (POI) for the selection of hotel, restaurant and UNESCO.

The options for the hotel are stars (from 2 to 5) and in case of restaurant will be fast food or fine dining. Once user selects the POI, the current location of the user will be calculated by the help of GPS and GPS coordinates will be saved in the mobile user. After the coordinates have been received by the mobile user, the POI and co-ordinates will be sent to the e-Torch server. The data will be processed in the e-Torch data repository and will be embedded in the XML data format that needs to be transmitted to the mobile user. Once the mobile device received the XML file, it will parse and display in another user screen that will display the list of POI located in current location of the user.

Figure 10 shows the main menu of the e-Torch mcommerce based GPS software for mobile client. The eTorch main menu presents the UNESCO, hotel, restaurant, map search functionalities for the client to choose. UNESCO site information option provides the 
location, background history, important details with pictures to highlight its importance. By selecting hotel information from the main menu, user will receive the nearest hotel information based on user's own GPS location. Same process will goes for the restaurant on selection. Finally, map search is simple Google application that downloads the map according to current GPS location of the user.

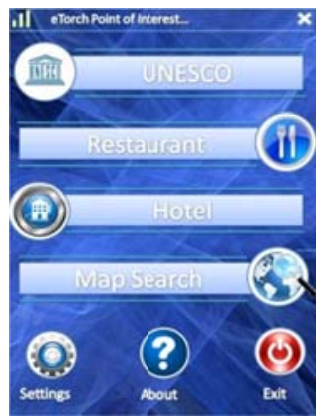

Fig. 10: e-Torch Main Menu (source: developed for this study)

\section{Database Repository layer}

Figure 11 represents the database modeling of the eTorch framework architecture that consists of eight tables. The names of the tables are identifier of the type of information stored within them.

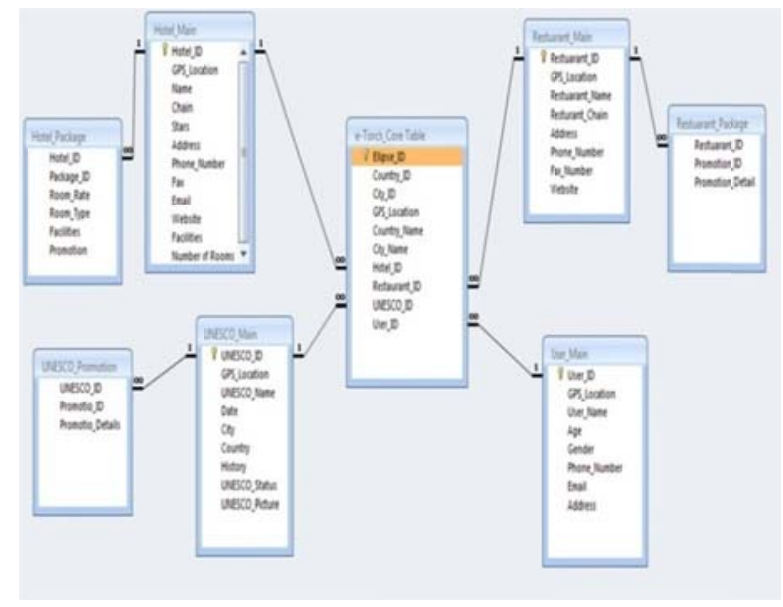

Fig. 11: The database diagram and relationship of the e-Torch Framework (source: developed for this study)

Main table is e-Torch Core table and other tables are subset of this core table with one-to-many relationship.
E-Torch Core table is the bridge between all the entities identified as building blocks of the proposed framework. The important fields of the core table are GPS location, User ID and places of interest such as hotel, restaurant and UNESCO sights, country and city name that facilitate to ellipses of proposed ellipse models. Each ellipse must have a unique ID; therefore, information related to each ellipse will be stored in our e-Torch database management system.

Tables related to hotels, restaurants and UNESCO sights contain all the information needed to be sent to the users and about their appropriate GPS location. With regards to the fact that the main feature of this research is location-based advertising, the most important parameter in User Main Table is GPS location of the user. The system needs to recognize the accurate position of the mobile end user and transfer locationbased information to them according to their interests.

\section{Communication Protocol}

Figure 12 illustrates the communication protocol of the e-Torch framework. The mobile device is connected with e-Torch server by sending a Connection (CON) message in order to be registered with the server. As a response, the e-Torch server will send the Acknowledgement (ACK) message to the mobile device after recognition of the client.

Users need to select the point of interests from the menu and send to the server with the current GPS location, therefore; GPS and POI (Point of Interests) message must be sent by mobile client to the server. On receiving the correct GPS location and the point of interest selected by the user, server must transmit another acknowledgement message to the mobile client for acknowledgement. During the GPS and POI message processing by server, Keep-alive (ALV) message will be sent in case of time out. Client will sent acknowledgement message on receiving the message again delivered by server.

After processing, server transfers related POI information to the client. Server will send POI information in XML format to the client. The size of XML file depends on the capacity of the POI advertising and promotion information. Once server send XML file, mobile client will respond by Acknowledgement message. If any XML files failed to get delivered to the mobile user, Enquire (ENQ) message will be sent by mobile client to server asking 
for retransmission of the file. On responding, server will verify the problem and send the XML file again. Client will acknowledge the correct XML file and server will send Disconnect (DIS) message and disconnect itself form network.

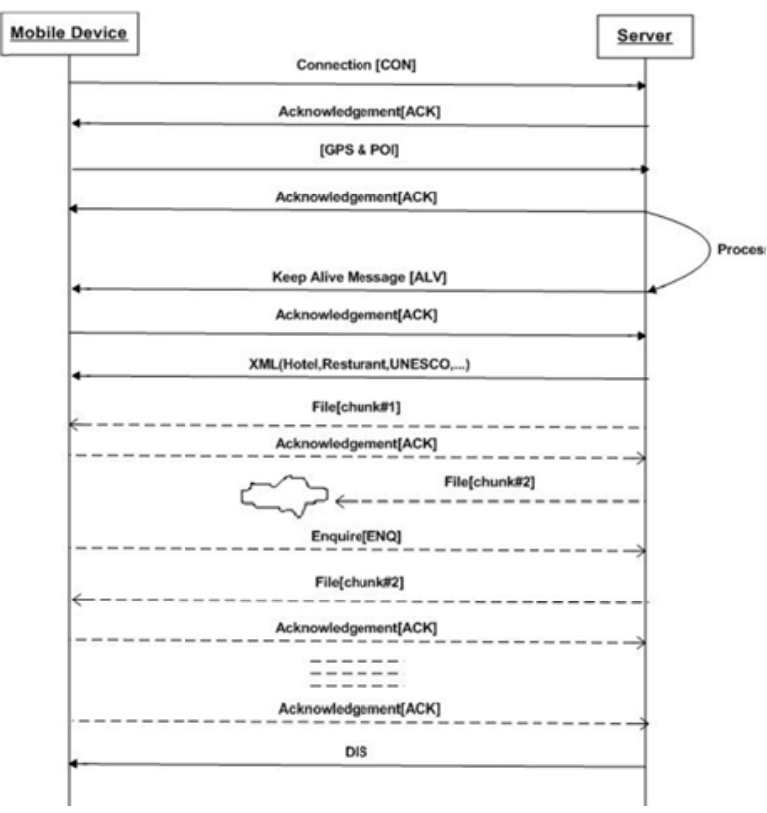

Fig. 12: The Communication Protocol of the e-Torch Framework (source: developed for this study)

\section{Algorithm of Our proposed Ellipse Model}

Our proposed ellipse model consists of the communication between the mobile client using either cellular network (GPRS/3G) or wireless network (WiFi) and the e-Torch server located remotely. There are two algorithms for our proposed e-Torch that run on mobile client and server side.

The algorithm of e-Torch on mobile side contains $\mathrm{S}$ (server socket), G (GPS location parameter), POI (Point of Interest) and Res (result) where $\mathrm{O}$ (output information from the server) that contains all the request information initiated by the mobile user. Algorithm begins with network initialization that connects with eTorch server by specifying IP address and port number. After the successful connection establishment, client will send point of interest (POI) and GPS location coordinates to the server. The server will process the information through e-Torch database management system, format the result in XML format, and send the information in the form of XML file. On receiving the XML file, the client will process the information and display the output in the mobile client screen (Table 1).

Table 1. e-Torch Mobile Side Algorithm (source: developed for this study).

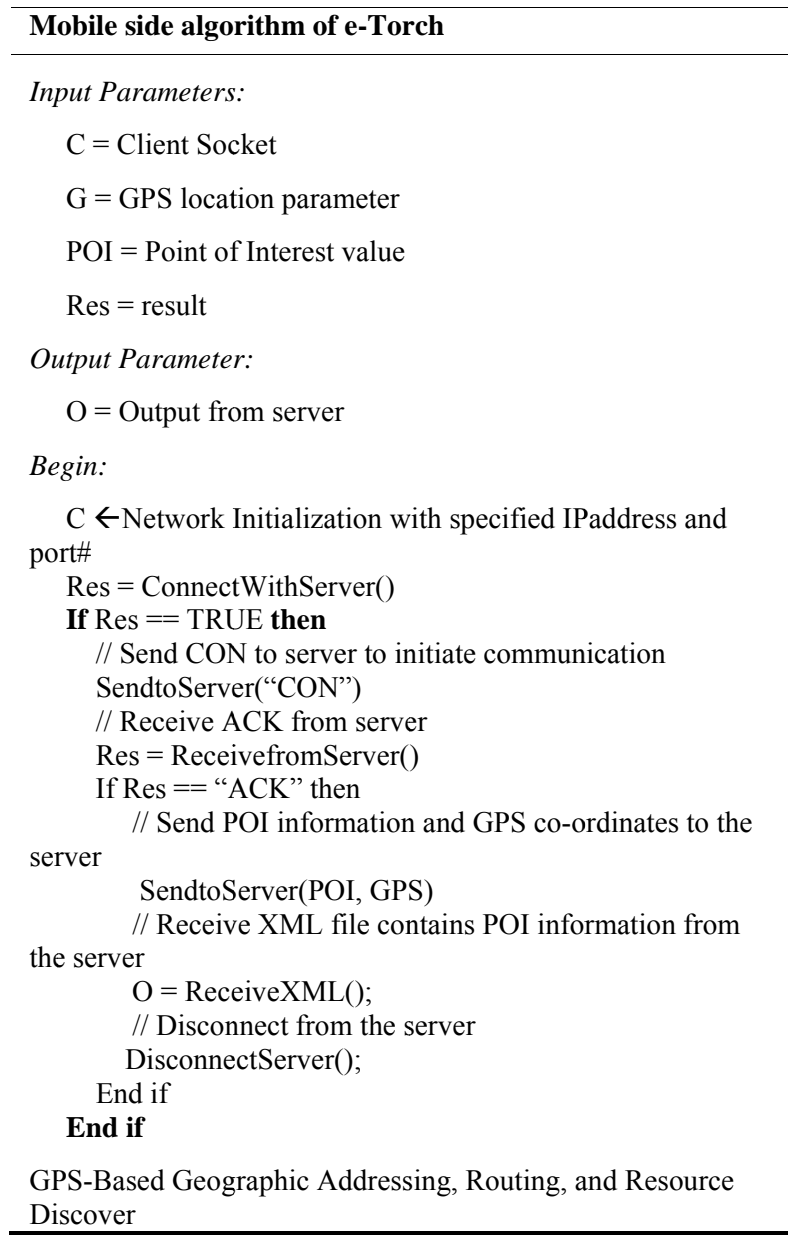

Similarly, in Table 2 we have discussed that the server is waiting for any client that will be connected to get the point of interest information from e-Torch database. Please note that the IP address of the server will be public that opens specific port number for the eTorch mobile client. Once the client connected to the eTorch server, the server will receive CON reserved message from the client showing the willingness to connect and request for the POI and GPS location. Once getting POI and GPS location co-ordinates from the eTorch mobile client, it will be immediately sent to the e- 
Torch server. e-Torch server will process the information and get back the required information about hotel or restaurant etc. After the query is been processed, it will be formatted in XML format and send back to the e-Torch client over the wireless or cellular communication medium. Once client receive the information, the client will send disconnect message to the e-Torch server that will disconnect the client and looking forward or waiting for another client to accommodate.

Table 2. E-Torch Server Side Algorithm (source: developed for this study).

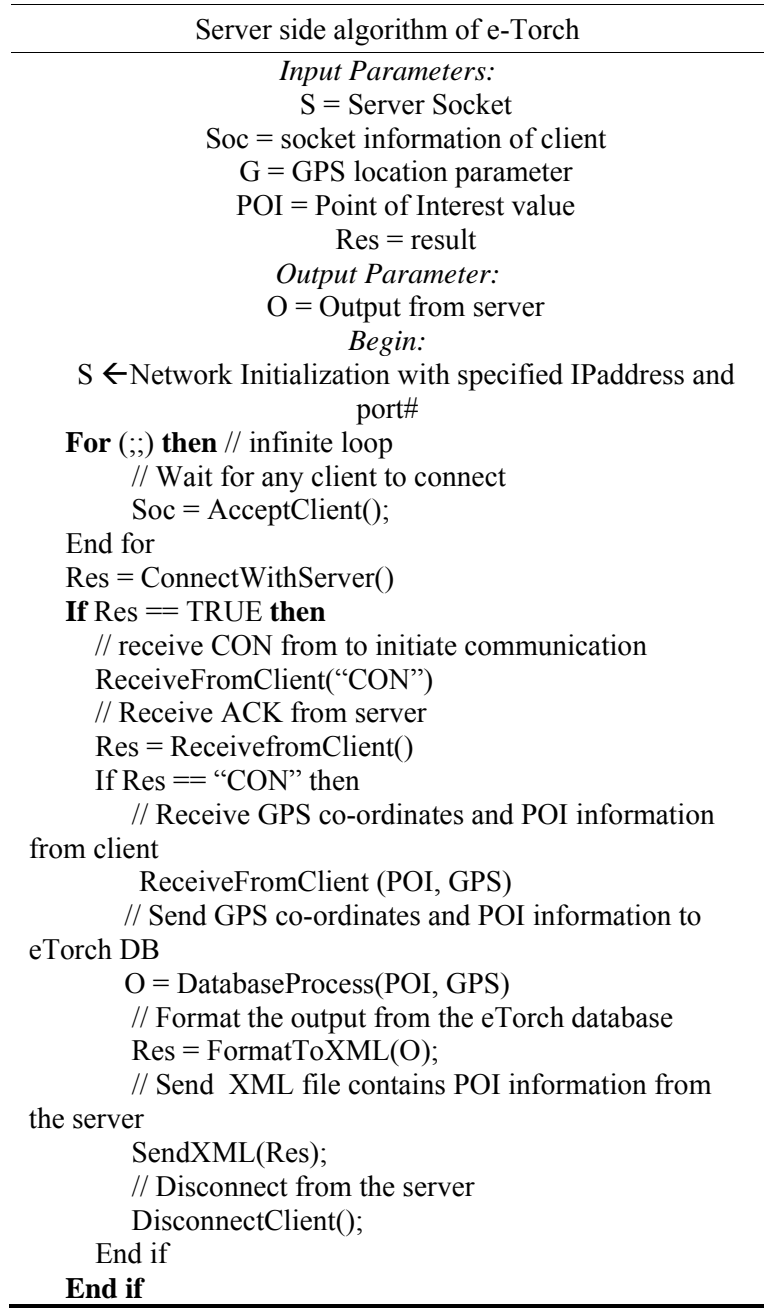

\subsection{Comparative Study on e-Torch with Other M- Commerce Module}

In the background section, we have reviewed different works proposed by researchers on GPS or GIS location tracking system that uses different communication technologies like Wi-Fi, GPRS, 3G/GSM etc. for transferring data from mobile location to the server. As shown in Table 3, each research proposes different model in order to address the efficiency and potential low cost for the tracking location and transferring the data from one location to another location efficiently and effectively. After deep and thorough review of the previous works, some weaknesses were discovered. The focus of this research is to propose a new model and framework that will able to address these issues.

Shibata, Terauchit, Kitanit, Yasumotot, Itot, and Higashinot (2006) proposed a method for real time traffic statistics collector that estimates the arrival time of vehicle at destination using inter-vehicle communication using wireless network and GPS. Their method entail dividing the area into segments of equal area that might save unnecessary information even for those areas that are not related to the experiments. The drawback leads to the in-efficiency of the framework and un-optimization of the performance. ${ }^{17}$

$\mathrm{Xu}$ and Yuan (2007) proposed acceptance model of mobile commerce with context and incentive consideration by using GPS-based taxi dispatching system. Their pilot study was incomplete where they did not measure the impact of context and incentive on Perceived Usefulness (PU) and Perceived Ease of Use (PEOU) and further more it did not addressed the technological impact. ${ }^{23}$

Neves, Ferreira, Esteves, Felix and Rodrigues (2008) proposed InHand an application model developed for local and remote use for context aware and locationaware using GPS technology. Although application model was proposed but it was not been mentioned how in real time it will be used especially when we talked about location tracking. ${ }^{37}$

Chadil, Russameesawang, Keeratiwintakorn (2008) proposes GPS tracking system known as Goo-Tracking comprises of GPS/GPRS module for location acquisition and message transmission. The tracking is through Google map server but there is no facility of mcommerce. In their future work, they propose to integrate the vehicle sensor with their system. ${ }^{12}$ 
Table 3. Comparative Study on E-Torch With Other MCommerce Module (source: developed for this study).

\begin{tabular}{|c|c|c|c|c|}
\hline Framework & Modeling & $\begin{array}{l}\text { Location } \\
\text { Tracking }\end{array}$ & $\begin{array}{l}\text { Communication } \\
\text { Technology }\end{array}$ & $\begin{array}{l}\text { POI } \\
\text { Point of } \\
\text { Interest }\end{array}$ \\
\hline $\begin{array}{c}\text { GPS-Based } \\
\text { Geographic } \\
\text { Adressing, } \\
\text { Routing, and } \\
\text { Resource } \\
\text { Discovery } \\
14\end{array}$ & $\begin{array}{l}\text { Addressing } \\
\text { Model, } \\
\text { Geometric } \\
\text { Routing }\end{array}$ & GPS & WiFi & No \\
\hline $\begin{array}{c}\text { Geographic } \\
\text { al Location } \\
\text { Tags on } \\
\text { Digital } \\
\text { Images } \\
38\end{array}$ & $\begin{array}{l}\text { Lightweight } \\
\text { Travelogue- } \\
\text { Authoring Tool }\end{array}$ & GIS & Internet & No \\
\hline $\begin{array}{c}\text { Traffic Jam } \\
\text { statistic } \\
\text { collector } \\
17\end{array}$ & $\begin{array}{c}\text { Inter-Vehicle } \\
\text { Communication }\end{array}$ & GPS & WiFi & No \\
\hline $\begin{array}{l}\text { Acceptance } \\
\text { model } \\
23\end{array}$ & $\begin{array}{c}\text { Context } \\
\qquad \& \\
\text { Incentive } \\
\text { Solution }\end{array}$ & GPS & WiFI & No \\
\hline $\begin{array}{c}\text { InHand } \\
37\end{array}$ & $\begin{array}{c}\text { Context and } \\
\text { location aware }\end{array}$ & GPS & $\mathrm{WiFi}, 3 \mathrm{G} / \mathrm{GSM}$ & No \\
\hline $\begin{array}{c}\text { Goo- } \\
\text { Tracking } \\
12\end{array}$ & $\begin{array}{l}\text { 2D-3D location } \\
\text { Tracking }\end{array}$ & GPS & GPRS & No \\
\hline $\begin{array}{c}\text { WPS \& Map } \\
\text { matching } \\
15\end{array}$ & $\begin{array}{c}\text { Centroid } \\
\& \\
\text { Fingerprinting }\end{array}$ & $\begin{array}{c}\text { WPS } \\
\text { with GPS }\end{array}$ & WiFi & No \\
\hline $\begin{array}{l}\text { Low-power } \\
\text { tracking } \\
\text { system } \\
11\end{array}$ & $\begin{array}{l}\text { GPS-GPRS } \\
\text { Module }\end{array}$ & GPS & GSM, GPRS & No \\
\hline $\begin{array}{l}\text { e-Torch M- } \\
\text { Commerce } \\
\text { Framework }\end{array}$ & Ellipse Model & GPS & GSM, GPRS, 3G & Yes \\
\hline
\end{tabular}

Athanasiou, Gerakakis, and Pfoser (2009) introduces Wireless Positioning System (WEP) based on 802.11 networks (Wi-Fi) in which they apply some WEP algorithms such as centroid and fingerprinting to derive the position of the object and map matching in order to improve the WPS accuracy. However, the wireless range is less and can have distortion if the users are more and the number of wireless equipments required to cover the geographical location is much more. Hence, the overall system can have an effect in case of fault in multiple wireless devices. ${ }^{15}$

Finally WPS \& Map matching low-power tracking system was presented by Wang, Zhao, $\mathrm{Xu}, \mathrm{Wu}$, and Luo (2010) that is web based application for tracking with the combination of Google Map and their tracking systems. They did not propose a model that can improve the relative information necessary to track location and then provide the architecture to exchange message communication. After reviewing these researches, we propose an architectural framework model that able to address the issues that have not been resolved and our research will take m-commerce as a consideration. ${ }^{11}$

In case of comparative study, we compare our eTorch framework with other M-Commerce based mobile advertising model and then justify that our framework is having more attributes such as transferring advertisement and promotion information related to points of interest (POI) to facilitate the end user. For the comparative study on e-Torch, we identify some important parameters like name, framework, modeling, location tracking, communication technology and point of interests (POI).

The scope of this research in terms of point of interest is limited to hotels, restaurant and UNESCO heritage sights. In Table 3 , we have make summarization of comparative study of our proposed eTorch M-Commerce based mobile advertisement system with literature of previous work that has been discussed in related work. The modeling attributes in Table 3 depict the method to locate the user as well as the information required to apply the relative methodology. The location tracking attribute shows the technology to track the mobile user where as the communication technology attribute shows the medium to send the request of the mobile user to the server. One of the important aspect of this research was the focus of $\mathrm{m}$-commerce based on the point of interests that is been used by mobile customers especially tourist. Our research work was based on the tracking of the mobile device and the location of the point of interest efficiently on the requested location through the ellipse model where the area is divided into different segments based on the geographical importance and the density of 
the number of POIs. The comparison of different researches along with our proposed framework is shown in Table 3 that presents framework name, modeling, location-tracking technique, communication technology and point of interests of the various works.

E-Torch framework presents ellipse model technique to map number of point of interests (POI) that includes hotel, restaurant, and UNESCO sights. The location of the mobile user is tracked by GPS and uses cellular network (GPRS and 3.5G) for the communication between e-Torch client and e-Torch framework. Moreover, e-Torch provides the facilities to get Point of Interests (POI) list that is been missing in the other framework covered extensively in other frameworks.

\section{Results and Discussion}

We conduct our experiments (Table 4) in $3 \mathrm{G}$ cellular network technology using notebook by categorizing them into in-door environment, outdoor environment (static) and out-door environment with mobility feature. E-Torch client sends its query regarding hotel, restaurant etc on $3 \mathrm{G}$ network connected with the remote E-Torch server having public IP address and port number.

We set the GPS location, therefore; when user sends the query to the E-Torch server via $3 \mathrm{G}$ networks, user's GPS location will be sent to the E-Torch server. The server will process the data information for the required hotel or restaurant based on the feature and geographical location co-ordinates obtained from GPS or A-GPS feature and gives the result in the form of XML file that is been received by our e-Torch client. The scope of our experiment was limited to sending the text information where voice and video will be for our future work. We conduct iterative number of experiments as presented in Table 4, in indoor, outdoor and mobility outdoor environment. We analyze that because of non-line of sight (NLOS) in the indoor environment, the time required to send and receive information is higher with approximately in seconds where as the outdoor static environment is much efficient with average round trip time (RTT) is in milliseconds. The most interesting part comes when we conducted experiment in a moving vehicle with mobility supports; the average rate fluctuates depending upon the distance of the base station or because of handover feature from one basestation to another base-station. In every case, the mobile user gets the result successfully.
Table 4. E-Torch RTT Experiment Results (source: developed for this study).

\begin{tabular}{|c|c|c|c|}
\hline Iteration & $\begin{array}{l}\text { Indoor E-Torch } \\
\text { RTT } \\
\text { Communication } \\
\text { (Milliseconds) }\end{array}$ & $\begin{array}{c}\text { Outdoor E- } \\
\text { Torch RTT } \\
\text { Communication } \\
\text { (Static) } \\
\text { (Milliseconds) }\end{array}$ & $\begin{array}{l}\text { Outdoor E-Torch } \\
\text { RTT } \\
\text { Communication } \\
\text { (Mobility) } \\
\text { (Milliseconds) }\end{array}$ \\
\hline 1 & 6158 & 718 & 1613 \\
\hline 2 & 5327 & 437 & 592 \\
\hline 3 & 1739 & 562 & 686 \\
\hline 4 & 4162 & 452 & 499 \\
\hline 5 & 1212 & 482 & 827 \\
\hline 6 & 8831 & 499 & 4431 \\
\hline 7 & 6856 & 624 & 8268 \\
\hline 8 & 1088 & 593 & 5226 \\
\hline 10 & 5807 & 578 & 577 \\
\hline 11 & 3940 & 624 & 796 \\
\hline 12 & 5175 & 686 & 6536 \\
\hline Average & 4191.25 & 521.25 & 2504.25 \\
\hline
\end{tabular}

\section{Conclusion}

Global Positioning System (GPS) based location tracking system is efficient way for tracking the exact co-ordinates of the mobile user to get the information about its current location. This mechanism is widely used in the m-commerce based advertisement system where the point of interest information about the hotel, restaurant, and shopping mall in specific location is sent to the mobile user based on the GPS location coordinates. Key findings, contribution to theory and practice, limitations, and future directions of this study are as follow:

\section{Key finding of this research:}

The main objective of this research is to propose GPS based system to facilitate location-based mobile advertisement and promotion system. We propose a location-based and m-commerce based e-Torch framework featuring point of interest such as hotel, restaurant, UNESCO sights etc. Furthermore, we also 
offer GPS based ellipse model for an efficient location based tracking system for points of interest for hotels, restaurant and UNESCO places. In order to gather requirement for e-Torch framework, we conducted an online survey to investigate proportion of GPS users in Penang as target market, to understand user's preference of Points of Interests (POI) and to estimate the price of $\mathrm{m}$-commerce advertising service for mobile GPS users.

\section{Contribution to theory and practice:}

The main contribution of this study to theory is the design of e-torch framework along with Ellipse model in order to improve location-based advertisement and promotion system. From the practice point of view, our online survey findings as well as our experiments that justify the success of the proposed system in in-door environment, outdoor environment (static) and out-door environment with mobility feature

\section{Limitations:}

We had some limitation for our experiments. One of those limitations was multimedia support, as we could not have video advertisement because of bandwidth constraints. The scope of our experiment was limited to sending the text information where voice and video will be for our future work. Next limitation was required good signals for cellular network; thus, the Quality of Service (QoS) is not guaranteed.

\section{Future Directions:}

From our investigations, we conclude that there is much potential of this work in other fields such as shopping mall, house and car rental, technology (mobile, laptop, etc.) promotions, find the location of friends and so forth that will open new horizons and broad target market in terms of scientific research. More enhancement and Improvements can be made to the mechanism of the ellipse modeling technique for cellular communication networks.

\section{References}

$1 \quad$ Mukesh Trehan and Ranju Trehan, Advertising and Sales Management (Rahul Jain V.K. (India) Enterprises; New Dehli, 2009).

2 Pantea Keikhosrokiani, 'E-Torch: Ellipse Model Framework for Location-Based and M-Commerce Advertising System', In: Dissertation for MSc IT (Universiti Sains Malaysia June 2011).

3 I. A. Getting, 'Perspective/navigation-The Global Positioning System', Spectrum, IEEE, Vol. 30, no. 12, 1993, pp. 36-8, 43-7.

4 Garmin, 'Garmin', (Garmin 2011).

5 Antuan Goodwin, 'Best 5 GPS navigation systems', (Samsung, Intel Products, AT\&T Products; 2010).

6 Foursquare, 'Foursquare ', (Foursquare 2011).

7 Cihan Cobanoglu, Silvia Ciccarelli, Robert R Nelson and Fred J DeMicco, 'Using Global Positioning Systems as a Marketing Tool: An Analysis of US Consumers' Use and Perceptions', Journal of Hospitality Marketing \& Management, Vol. 19, no. 6, pp. 556-74.

8 Bradford W. Parkinson and James J. Spilker Jr. (eds.), Global Positioning System: Theory and Application, Volume I (American Institute of Aeronautics and Astronautics, Inc.; 1996).

9 Ahmed EI-Rabbany, Introduction to GPS The Global Positioning System (Artech House Publishers; Boston, 2002).

10 M. Kennedy, The global positioning system and GIS: an introduction (Taylor \& Francis; 2002).

11 Wang Peng, Zhao Zhiwen, Xu Chongbin, Wu Zushun and Luo Yi, 'Design and implementation of the lowpower tracking system based on GPS-GPRS module', In: Industrial Electronics and Applications (ICIEA), 2010 the 5th IEEE Conference on. 2010), pp. 207-10.

12 N. Chadil, A. Russameesawang and P. Keeratiwintakorn, 'Real-time tracking management system using GPS, GPRS and Google earth', In: Electrical Engineering/Electronics, Computer, Telecommunications and Information Technology, 2008. ECTI-CON 2008. 5th International Conference on. 2008), pp. 393-6.

13 Ayad M. H. Khalel, 'Position Location Techniques in Wireless Communication Systems', In: Electrical Engineering Emphasis on Telecommunications. (Blekinge Institute of Technology Karlskrona,SWEDEN, 2010), p. 53.

14 T. Imielinski and J. C. Navas, 'GPS-Based Geographic Addressing, Routing, and Resource Discovery', Communications of the ACM, Vol. 42, no. 4, 1999, pp. 86-92.

15 Nikos Mamoulis, Thomas Seidl, TorbenBach Pedersen, Kristian Torp, Ira Assent, Spiros Athanasiou, Panos Georgantas, George Gerakakis and Dieter Pfoser, 'Utilizing Wireless Positioning as a Tracking Data Source', In: Advances 
in Spatial and Temporal Databases. (Springer Berlin Heidelberg; 2009), pp. 171-88.

16 Massimo Ficco and Stefano Russo, 'A hybrid positioning system for technology-independent location-aware computing', Software: Practice and Experience, Vol. 39, no. 13, 2009, pp. 1095-125.

17 N. Shibata, T. Terauchi, T. Kitani, K. Yasumoto, M. Ito and T. Higashino, 'A Method for Sharing Traffic Jam Information using Inter-Vehicle Communication', In: Mobile and Ubiquitous Systems: Networking \& Services, 2006 Third Annual International Conference on. 2006), pp. 1-7.

18 Jochen Schiller and Agnes Voisard, Location-Based Services (Morgan Kaufmann Publishers-Elsevier; 2004).

19 Kirsi Virrantaus, Jouni Markkula, Artem Garmash, Vagan Y. Terziyan, Jari Veijalainen, Artem Katasonov and Henry Tirri, 'Developing GIS-Supported Location-Based Services', In: Web Information Systems Engineering, 2001. Proceedings of the Second International Conference on. 2001), pp. 66-75, vol 2.

20 GregoryD Abowd, Barry Brumitt, Steven Shafer, Fredrik Espinoza, Per Persson, Anna Sandin, Hanna Nyström, Elenor Cacciatore and Markus Bylund, 'GeoNotes: Social and Navigational Aspects of Location-Based Information Systems', In: Ubicomp 2001: Ubiquitous Computing. (Springer Berlin Heidelberg; 2001), pp. 2-17.

21 Ling Zhao, Yaobin $\mathrm{Lu}$ and Sumeet Gupta, 'Disclosure Intention of Location-Related Information in Location-Based Social Network Services', International Journal of Electronic Commerce, Vol. 16, no. 4, 2012.

22 Lara Khansa, Christopher W. Zobel and Guillermo Goicochea, 'Creating a Taxonomy for Mobile Commerce Innovations Using Social Network and Cluster Analyses', International Journal of Electronic Commerce, Vol. 16, no. 4, 2012.

$23 \mathrm{Xu}$ Zhengchuan and Yuan Yufei, 'What is the Influence of Context and Incentive on Mobile Commerce Adoption? A Case study of a GPS-based Taxi Dispatching System', In: Management of Mobile Business, 2007. ICMB 2007. International Conference on the. 2007), p. 4.

24 Judy Strauss and Raymond Frost, E-Marketing (Pearson Education International; 2009).

25 Wil M.P. van der Aalst, 'TomTom for Business Process Management (TomTom4BPM) ', Springer, Vol. 5565/2009, 2009.

26 Andreas M. Kaplan, 'If you love something, let it go mobile: Mobile marketing and mobile social media $4 \times 4$, Business Horizons, Vol. 55, no. 2, 2012/4//, pp. 129-39.

27 Matthew W. Wilson, 'Location-based services, conspicuous mobility, and the location-aware future', Geoforum, Vol. 43, no. 6, 2012, pp. 1266-75.

28 Wan-Shiou Yang, Hung-Chi Cheng and Jia-Ben Dia, 'A location-aware recommender system for mobile shopping environments', Expert Systems with Applications, Vol. 34, no. 1, 2008, pp. 437-45.

29 P. D. Mzila, M. O. Adigun and S. S. Xulu, 'Service Supplier Infrastructure for Location-based M-Commerce', In: Internet Monitoring and Protection, 2007. ICIMP 2007. Second International Conference on. 2007), p. 35.

30 C. McTavish and S. Sankaranarayanan, 'Intelligent agent based hotel search \&amp; booking system', In: Electro/Information Technology (EIT), 2010 IEEE International Conference on. pp. 1-6.

31 Wan Zheng, 'Personalized Tourism Information System in Mobile Commerce', In: Management of eCommerce and e-Government, 2009. ICMECG '09. International Conference on. 2009), pp. 387-91.

32 W. Husain, Dih Lam Yih, Yen Heng Foo and N. Jothi, 'MyTourGuide.com: A Framework of a Location-Based services for tourism industry', In: Computer \& Information Science (ICCIS), 2012 International Conference on. pp. 184-9. 33 Runhe Huang, AliA Ghorbani, Gabriella Pasi, Takahira Yamaguchi, NeilY Yen, Beijing Jin, Ranieri Baraglia, Claudio Frattari, Cristinaloana Muntean, FrancoMaria Nardini and Fabrizio Silvestri, 'A TrajectoryBased Recommender System for Tourism', In: Active Media Technology. (Springer Berlin Heidelberg, pp. 196-205.

34 O. Rashid, P. Coulton and R. Edwards, 'Implementing location based information/advertising for existing mobile phone users in indoor/urban environments', In: Mobile Business, 2005. ICMB 2005. International Conference on. 2005), pp. 377-83.

35 Kai $\mathrm{Li}$ and Timon C. Du, 'Building a targeted mobile advertising system for location-based services', Decision Support Systems, Vol. 54, no. 1, 2012, pp. 1-8.

36 Pantea Keikhosrokiani, Norlia Mustaffa, MuhammadImran Sarwar, Amin Kianpisheh, Faten Damanhoori and Nasriah Zakaria, 'A Study towards Proposing GPS-Based Mobile Advertisement Service', In: Informatics Engineering and Information Science. (Springer Berlin Heidelberg; 2011), pp. 527-44.

37 Paulo A. C. S. Neves, David J. M. Ferreira, Daniel Esteves, Daniel R. M. Felix and Joel J. P. C. Rodrigues, 'InHand - Mobile Professional Context and Location Aware Tool', In: Software, Telecommunications and Computer Networks, 2008. SoftCOM 2008. 16th International Conference on. 2008), pp. 80-4.

38 K. Toyama, R. Logan, A. Roseway and P. Anandan, 'Geographic location tags on digital images', In: 11th ACM International Conference on Multimedia, MM'03. Berkeley, 2003), pp. 156-66. 